\title{
L'androïde chirurgien n'est pas encore né..., mais le chirurgien peut être assisté par un ordinateur
}

\author{
The Android Surgeon Is yet to Be Born..., but Surgeons Can Be Helped by a Computer
}

\author{
F. Guillemin \\ (C) Lavoisier SAS 2016
}

Ce numéro spécial consacré à la chirurgie robotique permet de faire un point sur le domaine particulier de la télécœeliochirurgie plus spécifiquement appliquée à la cancérologie. L'histoire des techniques chirurgicales montre les progrès réalisés et le futur qui reste à inventer.

La cœlioscopie remonte au début du siècle, et la chirurgie coelioscopique date du milieu des années 1980, elle fut qualifiée « the Second French Revolution ». Il y eut des idées futuristes pertinentes, mais sans développement industriel (Fig. 1). Les procédures cœliochirurgicales se sont alors multipliées dans toutes les spécialités, diversifiées et perfectionnées (One-Port, NOTES...). La chirurgie mini-invasive était inventée et devenait quasiment un standard dans toutes les spécialités. Les progrès principaux sont liés à la qualité de l'image, désormais en haute résolution et stéréoscopique, et à quelques outils ancillaires.

Le début des années 2000 voit la mise au point de la télécœliochirurgie, appelée par abus de langage le robot chirurgical, dont l'essor commercial est spectaculaire. La télécœliochirurgie est un système " maître-esclave » où les mouvements du chirurgien sont fidèlement transmis aux bras effecteurs (dits bras passifs à contraintes dynamiques). L'utilisation est simple et intuitive. Les défis de mise au point d'une telle machine sont importants dans le domaine de l'optique et de l'électronique pour le rendu 3D d'une image de grande qualité et dans le domaine de la mécanique pour donner aux instruments chirurgicaux les sept degrés de liberté nécessaires, qu'ils soient introduits par des trocarts différents ou par un port unique. L'ergonomie pour le chirurgien est un bénéfice majeur, atténuant la fatigue et le risque de troubles musculosquelettiques et permettant ainsi la réalisation de procédures complexes, a priori dans de meilleures conditions

F. Guillemin $(\bowtie)$

Institut de cancérologie Jean-Godinot, centre de lutte

contre le cancer de Reims, Champagne-Ardenne,

1, rue du Général-Kœnig, CS 80014 ,

F-51726 Reims cedex, France

e-mail : francois.guillemin@reims.unicancer.fr de sécurité. Ce concept, rétablissant l'axe « œil-mainspieds » et dissociant la place et la position du chirurgien par rapport au site opératoire, sans préjudice pour le patient puisque c'est la machine qui s'adapte au patient, est un vrai progrès qui ne pourra plus être remis en cause.

Aujourd'hui, ce qui devient réellement intéressant c'est l'intégration d'automates, c'est-à-dire de systèmes autorégulés fonctionnant en circuit fermé dans lesquels une information en entrée va conditionner une action en sortie. Ils assurent une fonction définie. Les pinces agrafeuses contrôlent la qualité de la prise (épaisseur et pression) avant d'autoriser l'agrafage motorisé. La thermofusion est un système d'hémostase vasculaire qui gère automatiquement le courant délivré en fonction de l'impédance tissulaire pour obtenir une coagulation-déshydratation sans carbonisation. Le positionnement des trocarts-instruments est proposé automatiquement par trigonométrie selon l'axe de la caméra qui vise le site sur lequel intervenir. L'asservissement de la position des bras de la machine aux mouvements de la table d'opération permet de conserver les axes de pénétration des trocarts sans nécessité de repositionnement de ceux-ci, ce qui élargit le champ des possibles en modifiant l'exposition du site opératoire. Le retour de force est une action d'automatique subtile dans sa réalisation technique ; plusieurs forces (pression, résistances mécaniques) sont mesurées aux mors de l'instrument, et ces données sont renvoyées aux « joysticks » modifiés en effecteurs agissant en retour sur les doigts et la main de l'opérateur.

Demain, c'est l'intégration d'automates plus complexes qui va faire évoluer la chirurgie mini-invasive. La navigation assistée par ordinateur ou « comanipulation » :

- réalise le recalage entre le modèle 3D du patient (ou de l'organe) construit à partir du scanner ou de l'IRM et le patient lui-même ;

- intègre en temps réel la position d'un instrument dont les déplacements sont contrôlés par le chirurgien dans une zone non accessible à la vue.

La réalité virtuelle augmentée, appliquée aux surfaces déformables et aux organes mobiles, superpose l'image 

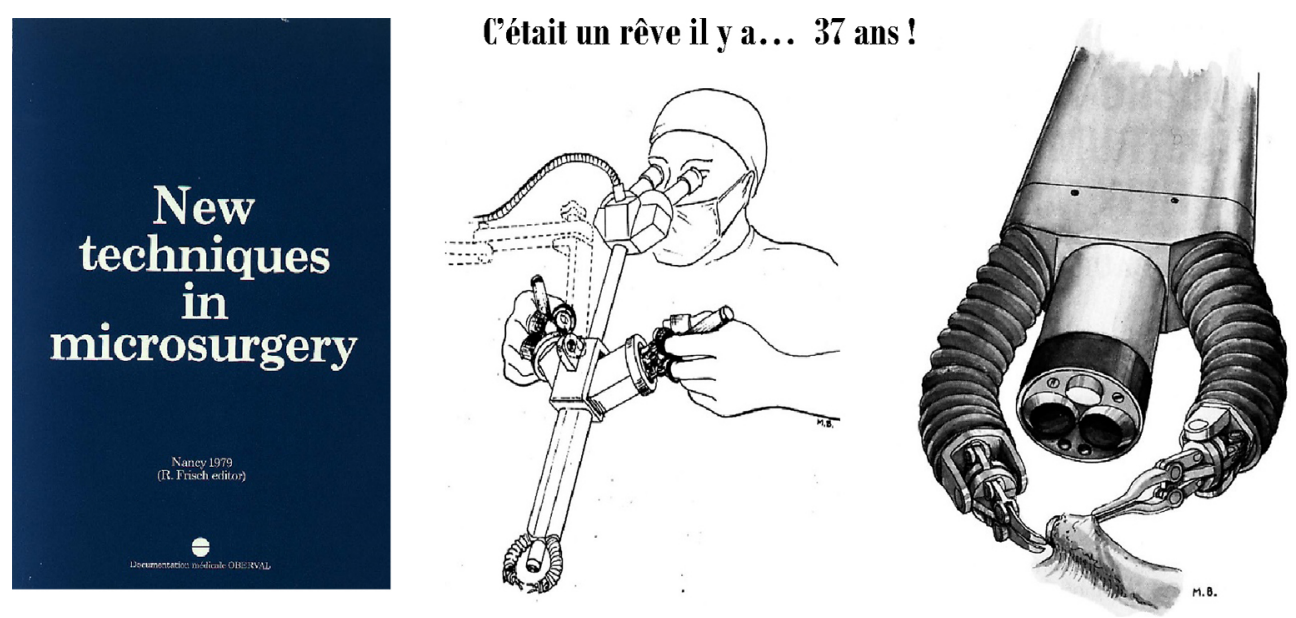

Fig. 1 Brice M, Guillemin F (1979) Considerations upon a new technological approach of problems in deep microsurgery

réelle de l'organe et l'image virtuelle reconstruite (par exemple : réseau vasculaire, tumeur) à partir d'un scanner ou d'une IRM de l'organe. Au-delà, on conçoit une chirurgie guidée qui contraint le déplacement en zone de sécurité, puis une automatisation complète de certains temps opératoires précis tels que déjà réalisés en neurochirurgie, en orthopédie ou en implantologie dentaire (sur des structures fixes non ou peu déformables), avec un suivi par imagerie embarquée temps réel. De ce point de vue, l'utilisation d'instruments miniaturisés, motorisés et asservis s'intégrera dans l'automatisation du geste.

Au décours de cet éditorial, se dessine le domaine transdisciplinaire de la « Surgétique » associant des technologies issues de l'informatique, du traitement d'images, de la mécanique, de l'automatique et de la robotique pour des applications chirurgicales afin d'étendre les capacités d'intervention dans un environnement complexe en préservant toutes les potentialités du geste et en augmentant les capacités de perception et d'actions. On y associe naturellement l'« Haptique » (interface homme-machine) dont le retour de force et la commande vocale sont de bons exemples. La « Cobotique » (domaine à l'interface de l'intervention humaine, de la Cognitique [traitement automatique de la connaissance], de la biomécanique et de la robotique) a pour objectif de développer des interfaces conviviales, intelligentes, transparentes, acceptables par le chirurgien et lui permettant de gérer un robot d'assistance chirurgicale.

La chirurgie mini-invasive et l'intégration de la robotique sont des évolutions inéluctables, et la télécœliochirurgie en est une des étapes. Les perspectives immédiates et à plus long terme sont passionnantes. Le coût financier est un paramètre limitant, sauf à trouver un modèle économique tolérable et à démontrer in fine un bénéfice pour les patients et les chirurgiens.

Nous soulignerons les progrès pour l'enseignement par des dispositifs de simulation de chirurgie interactifs. L'apprentissage du geste se fait par une immersion en environnement virtuel qui restitue les conditions de l'opération réelle. C'est devenu une des composantes obligatoires de la formation des internes en chirurgie.

Le concept de chirurgie assistée par ordinateur présente plusieurs niveaux d'assistance au geste (navigation, guidage, téléchirurgie) jusqu'à une autonomie complète. La parfaite connaissance de l'environnement et la supervision humaine resteront encore indispensables... et pour longtemps ! 